\title{
Torque in Nested Halbach Cylinders
}

\author{
T. R. Ní Mhóocháin ${ }^{1,2}$, J. M. D. Coey ${ }^{1}$, D. L. Weaire ${ }^{1}$, S. M. McMurry ${ }^{1}$ \\ ${ }^{1}$ Trinity College, Dublin 2, Ireland \\ ${ }^{2}$ Magnetic Solutions Ltd, Unit 13, IDA Centre, Pearse St., Dublin 2, Ireland.
}

\begin{abstract}
Permanent magnet variable flux sources are produced by rotating two nested $N$-segment, permanent magnet, Halbach cylinders. Torque is primarily an end effect, determined by field non-uniformities due to the finite length and segmented structure of the cylinders. Due to symmetry, one cylinder experiences zero torque in a uniform applied field. Experiment and numerical simulation reveal that the torque is composed of a dominant term of frequency $1 /(2 \pi)$ and harmonics at $(k N \pm 1) /(2 \pi)$, where $k$ is an integer.
\end{abstract}

Index Terms- Permanent magnet variable flux sources, Halbach cylinders, torque, surface charge method, motors.

\section{INTRODUCTION}

Permanent magnet variable flux sources are finding increasing application in markets traditionally dominated by the electromagnet. They can provide high, uniform, localised magnetic fields up to $2 \mathrm{~T}$, while being smaller and lighter than their electrical counterparts [1]. They require no special power supply or cooling unit, and can be designed to produce close to zero stray field outside a region of interest. Many geometries have been explored [2] - [4]. Applications range from laboratory instrumentation to the manufacture of magnetic thin film devices to $\mathrm{NMR}$ imaging.

Halbach cylinders, or dipole rings, have particularly general and wide-ranging applicability. The ideal design [4] is shown in Fig. 1(a). The magnitude of magnetisation is constant, while its orientation varies continuously: at an angular position $\phi$ in the cylinder, measured clockwise from the $y$-axis, the magnetisation has orientation $2 \phi$. An infinitely long cylinder produces a uniform magnetic field in the $y$-direction within the bore, and zero field everywhere outside the cylinder.

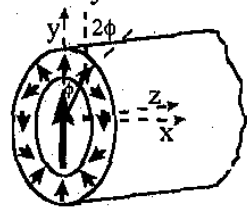

(a)

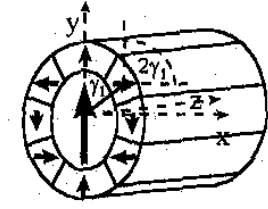

(b)

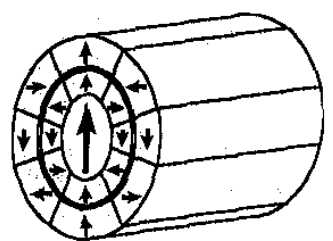

(c)

Fig.1: (a) the ideal, infinitely long design, with uniformly varying magnetisation; (b) finite length approximation to (a) using 8 segments; (c) nested cylinders can produce varying fields in their central bore.

T. R. Ní Mhíocháin - treasa@magnetic-solutions.com

This work was partly supported by Forbairt and the U.S. Navy, contract number ONR N00014-96-1-0767.

Manusoript submitted for review $1^{\text {st }}$ March 1999.
Finite length approximations to this design [5] are constructed from a discrete number $N$ of segments, typically eight, as shown in Fig.1(b). Variable flux sources are developed by nesting two such cylinders [3], and rotating them relative to each other as in Fig. 1(c) so that the field in the bore is the vector sum of the two individual fields.

Torque between the cylinders as they rotate is an important consideration when attempting to implement this design. Here we present results of experiment and numerical simulation of torque in nested systems, and show that a direct relationship exists between torque and the finite length and segmented structure of these devices.

\section{EXPERIMENTAL RESULTS}

We measured the variation in torque as a function of $\alpha$ for a two cylinder nested system, where $\alpha$ is the angle through which the inner cylinder is rotated from the position where its magnetisation is fully aligned with that of the outer. The cylinder dimensions are given in Table I.

TABLE I

DIMENSIONS OF MAGNETIC CYLINDERS

$\begin{array}{lcc} & \text { Inner Cylinder } & \text { Outer Cylinder } \\ \text { Inner Radius (mm) } & 26 & 52.5 \\ \text { Outer Radius (mm) } & 47.5 & 110 \\ \text { Length (mm) } & 100 & 100 \\ \text { Segment Number N } & 8 & 8\end{array}$

Segment Number $N$

Each cylinder was connected, via a system of gears, to a d.c. motor. The gearing system comprised a gearbox and a worm wheel assembly. The position of each cylinder at any moment of time was recorded via an angular position encoder.

The torque $\Gamma$ generated by a d.c. motor is directly proportional to $I$, the current it draws,

$$
\Gamma=k_{T} I
$$

where $k_{T}$ is a torque constant. For these motors, operating at low frequency of rotation of the motor head (as is the case here), $k_{T}$ is given by

$$
k_{T}=(V-I R) / \omega
$$

where $V$ is the operating voltage, $R$ is the resistance at the motor terminals and $\omega$ is the angilar speed of the rotor in radians/s.

We run the motor at constant voltage and monitor variations in current and motor speed. From these data we calculate the torque generated by the motor at any angle $\alpha$. This is then related to the torque experienced by the cylinder via

$$
\Gamma=\Gamma_{\text {mot }} r_{\mathrm{gb}}\left(n_{\text {teeth }} / n_{\text {start }}\right) \eta_{\mathrm{in}} \eta_{\mathrm{ww}} \eta_{\mathrm{gb}}
$$

where $r_{\mathrm{gb}}$ is the gearbox ratio, $n_{\text {teeth }}$ the number of teeth in the worm wheel and $n_{\text {start }}$ the number of starts, $\eta_{m,}, \eta_{w w}$ and $\eta_{g b}$ are the motor, worm-wheel and gearbox efficiencies respectively.

For a constant applied voltage of $12 \mathrm{~V}$, Fig.2(a) plots the torque experienced by the cylinder as a function of $\alpha$ for two full. rotations of the inner cylinder. Here the cylinder is rotating in the clockwise direction. 
The variation in torque is essentially sinusoidal around one full rotation of the cylinder, as will become clear in Section III. For one half of each cycle, the motor is working to overcome the magnetic torque, so that an increase in load torque is reflected by an increase in the current drawn by the motor and a decrease in the speed, and vice versa.
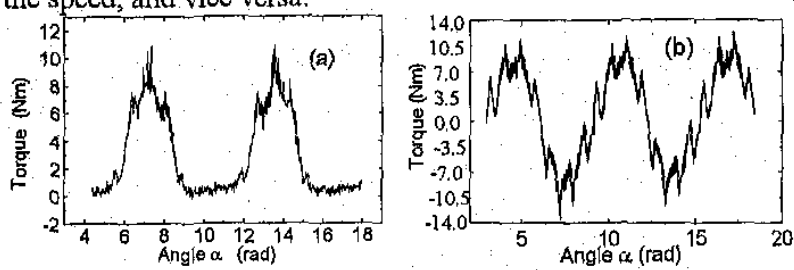

Fig.2: (a) Torque measured on the inner cylinder as it is rotated clockwise through angle $\alpha$ in the bore of the static outer cylinder; (b) torque over 2.5 rotations of the inner deduced from both clockwise and anticlockwise rotation

For the second half of each cycle, both the motor and the magnetic torque rotate the cylinder in the same direction. So there is effeotively zero load on the motor. The current drawn is reduced and the speed increased. However, the maximum (no load) speed $n_{0}$ and the minimum (no load) current $I_{0}$ are determined by the applied voltage and the terminal resistance of the motor, and their values are defined for a given motor. Hence, in this region of the graph, both current and speed remain approximately constant.

Similar data were collected for rotation in the anticlockwise direction. These two sets of data were combined to indicate the structure of the torque variation over 360 degree cycles (in the process, the absolute values were shifted downwards by a constant value), The combined data for the torque variation over 2.5 full rotations of the inner cylinder are shown in Fig.2(b).

Fig. 3 shows a Fourier transform of the data in Fig.2(b). Amplitudes of the Fourier coefficients for torque are plotted against frequency. The dominant term is evident at $f=1 /(2 \pi)$, indicating that the variation is mainly sinusoidal. Higher harmonics of this fundamental are also evident, in particular at $7 f, 15 f$ and $23 f$, each with a smaller neighbouring peak around $9 f, 17 f$ and $25 f$ respectively. Thus harmonics occur at $(k N \pm 1) f$, where $N=8$ is the number of segments in the cylinders.

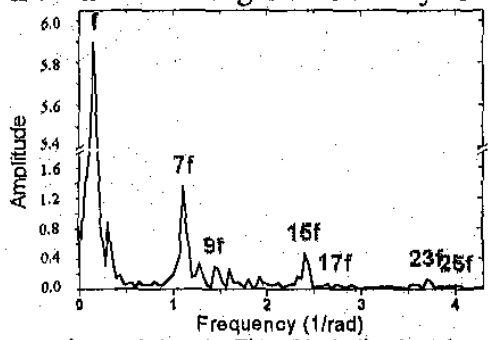

Fig.3 : Fourier transform of data in Fig.2(b), indicating the main frequency variations of current with angle as the inner cylinder rotates.

\section{NUMERICAL MODEL}

Our numerical simulation has two stages. Firstly we calculate the field in the bore of the outer cylinder; secondly we calculate the torque experienced by the inner cylinder in this field.

1) Field Calculation: Our method of field calculation is based on the assumption that each segment has a perfectly rigid polarisation $J$. The magnetic induction within a block of magnetic material is $\mathbf{B}=\mu_{0} \mathbf{H}+\mathbf{J}$. In a magnetostatic system, $\nabla \times \mathbf{H}=0$, so we may define a scalar potential $\Phi$ by $\mathbf{H}=-\nabla \Phi$. Given also that $\nabla \cdot \mathbf{B}=0$, then, $\nabla \cdot \mathbf{H}=-\nabla^{2} \Phi=-(\nabla . J) / \mu_{0}$. So that $\Phi$ satisfies a Poisson equation with magnetic charge density. $\rho=-\nabla . J$. The solution to this Poisson equation is given in terms of volume and surface charge densities [6].

In the case of a segmented cylinder, however; each segment is assumed to have uniform and perfectly rigid $J$. The volume magnetic charge density $p$ in each segment is zero, $\rho=-\nabla . \mathbf{J}=0$, so the magnet can be represented by charged surfaces, each with surface magnetic charge density $\sigma$ given by $\sigma=\mathbf{J} \cdot \mathbf{n}$, where $\mathbf{n}$ is the unit normal to the surface. Analytical expressions for the vector field at a point, due to a charged rectangular surface, have been derived by Akoun and Yonnet [7]. A segmented cylindrical. magnet is a combination of prisms. The total field at any point is simply the sum of the field contributions from each face.

On a cross-section of the outer cylinder, we specify a grid of points, each with co-ordinates $\mathbf{r} \equiv(x, y, z)$. At each of these points we calculate the components of the field $H_{\mathrm{x}}, H_{\mathrm{y}}$ and $H_{\mathrm{z}}$. The field varies along the length of the cylinder as well as across the widttr of the bore, and so is calculated across similar grids on a number: of different, equally spaced planes. Each point at which the field is calculated, is surrounded by an element of material with volume $\Delta V$.

2) Torque Calculation: The total torque $\Gamma$ experienced by the inner cylinder is calculated by summing the torques $\Gamma^{(i)}$ on the volume elements $\Delta V^{(i)}$ of the inner cylinder, due to the field of the outer cylinder,

$$
\Gamma=\sum_{i} \Gamma^{(i)}=\sum_{i} \Delta V \mathbf{J}^{(i)} \times \mathbf{H}^{(i)}+\mathbf{r}^{(i)} \times \mathbf{F}^{(i)}
$$

where at a point $\mathbf{r}^{(i)}$ in the inner cylinder, $\mathbf{H}^{(i)}$ is the field due to the outer cylinder, $\mathrm{J}^{(i)}$ is the polarisation of the inner cylinder, and the force $\mathrm{F}^{(\mathrm{i})}$ due to the field gradient is found numerically from

$$
\mathbf{F}^{(i)}=-(\nabla E)=\nabla\left(\mathbf{J}^{(i)} \cdot \mathbf{H}^{(i)}\right) \Delta V^{(i)} \text {. }
$$

Symmetry of the system implies zero values for $\Gamma_{x}$ and $\Gamma_{y}$, so that the only non-zero component of torque is $\Gamma_{2}$, about the axis of the cylinder. This is confirmed by calculation.

3) Numerical Results; Fig. 4 shows $\Gamma_{\mathrm{z}}$ as a function of $\alpha$.

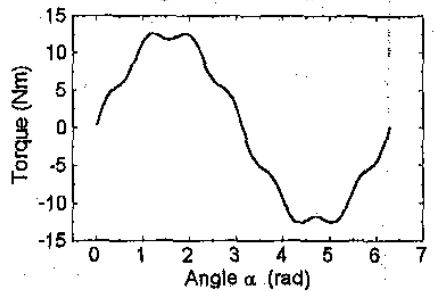

Fig. 4 : Calculated torque on inner cylinder as a function of $\alpha$.

The fundamental variation in $\Gamma_{\mathrm{z}}$ goes as $\Gamma_{z 0}=A \sin \alpha$. In addition, $\Gamma$ is modulated by structure associated with segmentation. Fig.5(a) shows a Fourier transform of the calculated torque.

The fundamental frequency $f^{\prime}=1 /(2 \pi)$ corresponds to the leading sine term. The $7^{\text {th }}$ harmonic is also strong. Higher harmonics are present, but with smaller amplitudes. To see these. more clearly, we fit a curve of the form $B \sin \alpha+C \sin (7 \alpha)$ to the data in Fig. 4 , where $B$ and $C$ are constants. The fit curve is then subtracted from the original data. In so doing we largely remove the $f$ and $7 f$ terms. A Fourier transform of the remaining data is shown in Fig.5(b). Higher harmonics can now be seen to occur. 
in pairs at $(N-1) f,(N+1) f,(2 N-1) f,(2 N+1) f$, etc., that is at $(k N \pm 1) f$, where $N=8$ is the number of segments and $k$ is an integer. These harmonics coincide with the frequencies found experimentally in Fig.3. The peak calculated value of $13 \mathrm{Nm}$ is larger than that observed experimentally due to the fact that the polarisation is not perfectly rigid.
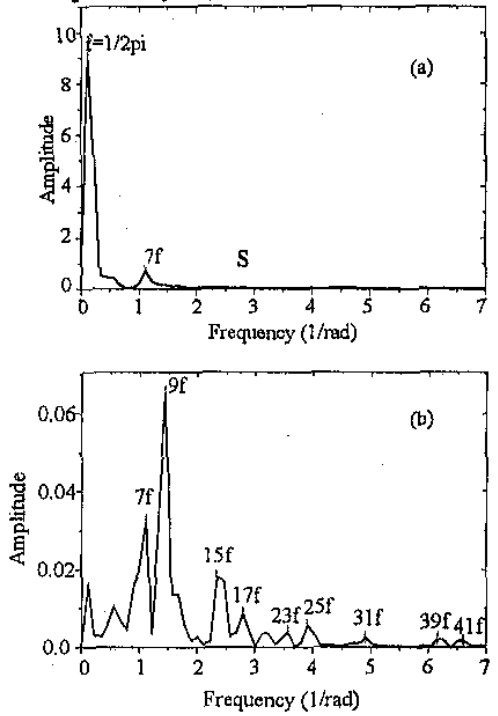

Fig.5: (a) Fourier transform of data in Fig.4; (b) without the $f$ and $7 f$ terms.

\section{ORIGIN OF THE TORQUE}

The Reciprocity Theorem states that the energy of one piece of magnetic material in the field of another is equal to the energy of the second in the field of the first. Thus both magnets must experience equal and opposite torques.

Consider now the case of two ideal, infinite length cylinders as in Fig. 1(a), nested concentrically. Both cylinders have completely uniform bore fields, and zero external stray fields. So the outer cylinder experiences zero applied field, and thus zero torque. By reciprocity, the inner cylinder also experiences zero torque. So there is zero torque on the inner cylinder in a constant applied field. Similarly, a segmented cylinder of even $N$, experiences zero torque in constant applied field as the torque on each segment is exactly cancelled by torque on another segment. This is confirmed numerically. The torque in our systems must therefore arise from the field non-uniformities due to their finite length and segmented structure:

Consider the field profile in the bore of a cylinder with the radial dimensions given Table I, calculated using the surface charge model. Fig.6(a) shows the profile of $B_{y}$ in the bore at the centre $(z=0)$ when the cylinder is very long and has a large number of segments $(N=128)$ to approximate the case of infinite length and uniformly varying magnetisation in Fig.1(a), $B_{y}$ remains constant at all points inside the bore as expected for an ideal cylinder. In a real cylinder, however, there are two main features in field non-uniformity:

1) Finite Length: Fig.6(b) shows the profile for a cylinder with length $100 \mathrm{~mm}$, and $N=128$ to approximate uniformly varying magnetisation.

The magnitude of the field at $\phi=0$ and $\phi=\pi$ is greater than that at $\phi=\pi / 2$ and $\phi=3 \pi / 2$. As the cylinder is made longer, the extent of this effect at the central $z=0$ position decreases, although it persists at both ends. This effect is therefore an end effect associated with the finite length of the cylinder. This is the cause of the leading sinusoidal variation in the torque.

2) Segmentation: Fig.6(c) shows the profile for an 8-segment, finite length cylinder. Again the end effect is apparent, but additional peaking in the field occurs near the boundaries between adjacent segments. Here there are 8 peaks. For a 16segment cylinder there would be 16 peaks and so on. This is the origin of the smaller, $N$ dependent, torque variation.

Numerical values of the contributions to the torque from different cross-sections along the axis in the cylinder, show that the main contributions come from the end regions. The total value of the torque is unchanged by increasing the length of the cylinder to larger, finite values, and the contribution from the central region becomes negligible. This indicates that the torque is primarily an end effect.

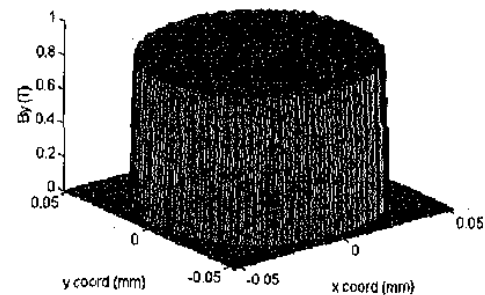

(a)

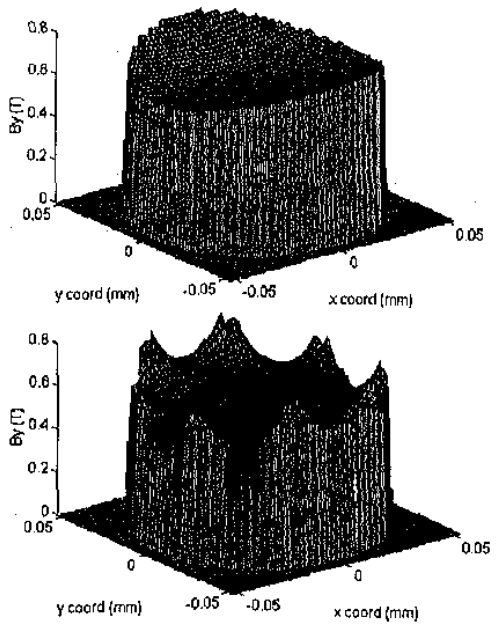

(b)

Fig.6: Field profile in the bore of (a) an infinitely long cylinder, with uniformly. varying magnetisation; (b) a finite length cylinder with uniformly varying magnetisation; (c) a finite length, 8-segment cylinder.

\section{ACKNOWLEDGMENT}

Particular thanks to Farid Bengrid and Laurent Clochard.

\section{REFERENCES}

[1] M, Coey and D. Weaire, Ind.Physicist, pp. 34-6, Sept. 1998.

[2] O. Cugat, P. Hansson and J. M. D. Coey, IEEE Trans. Magn., vol. 30, no. 6, pp, 4602-4, 1994

[3] H. A. Leupold, E. Potenzianni II and M. G. Abele, J.Appl.Phys., vol. 64, no, 10, pp, 5994-6, 1988.

[4] H. A. Leupold in Rare Earth Permanent Magnets, J. M. D. Coey (editor), 1996

[5] K. Halbach, Nucl.Instr.and Meth., vol. 169, pp. 1-10, 1980.

[6] J. D. Jackson, Classical Electrodynamics, Wiley, 1962.

[7] G. Akoun and J. P. Yonnet, IEEE Trans. Magn, vol. 20, 1964-4, 1984. 